\title{
Far infrared spectroscopy on the three-dimensional dilute antiferromagnet $\mathbf{F e}_{x} \mathbf{Z n}_{1-x} \mathbf{F}_{2}$
}

\author{
J. Satooka and K. Katsumata \\ RIKEN (The Institute of Physical and Chemical Research), Wako, Saitama 351-0198, Japan \\ D. P. Belanger \\ Department of Physics, University of California, Santa Cruz, California 95064
}

(November 20, 2018)

\begin{abstract}
Fourier-transform Infrared (FT-IR) Spectroscopy measurements have been performed on the three-dimensional dilute antiferromagnet $\mathrm{Fe}_{x} \mathrm{Zn}_{1-x} \mathrm{~F}_{2}$ with $x=0.99 \sim 0.58$ in far infrared (FIR) region. The FIR spectra are analyzed taking into account the ligand field and the local exchange interaction probability with $J_{1} \sim J_{3} ;\left|J_{1}\right|,\left|J_{3}\right| \ll\left|J_{2}\right|$, where $J_{1}, J_{2}$ and $J_{3}$ are the nearest neighbor, second nearest neighbor and third nearest neighbor exchange interaction constants, respectively. The concentration dependence of the FIR spectra at low temperature is qualitatively well reproduced by our analysis, though some detailed structure remains unexplained.
\end{abstract}




\section{INTRODUCTION}

The dilute antiferromagnet $\mathrm{Fe}_{x} \mathrm{Zn}_{1-x} \mathrm{~F}_{2}$ has been studied extensively over the last two

decades. In the concentrated region with $x>0.4, \mathrm{Fe}_{x} \mathrm{Zn}_{1-x} \mathrm{~F}_{2}$ is an ideal example of the random-exchange Ising model without an applied field, and it becomes a prototypical example of the random-field Ising model when the field is applied along the spin easy axis [1]. Near and below the percolation threshold $x_{p} \sim 0.24$ [2], $\mathrm{Fe}_{x} \mathrm{Zn}_{1-x} \mathrm{~F}_{2}$ shows a cluster-glass behavior [3, 4]. Paduani et al. have investigated the magnetic excitations in $\mathrm{Fe}_{x} \mathrm{Zn}_{1-x} \mathrm{~F}_{2}$ using inelastic neutron scattering techniques and have reported that the spectrum obtained at a low temperature can be reproduced with three Gaussian peaks [5]. No theoretical explanation has been given for this. In order to clarify the nature of the magnetic excitations in $\mathrm{Fe}_{x} \mathrm{Zn}_{1-x} \mathrm{~F}_{2}$, we have investigated the far infrared (FIR) spectra in $\mathrm{Fe}_{x} \mathrm{Zn}_{1-x} \mathrm{~F}_{2}$ using a Fourier-transform Infrared (FT-IR) spectrophotometer with high resolution in zero field and tried to understand the results.

\section{EXPERIMENTAL DETAILS}

The single crystals of $\mathrm{Fe}_{x} \mathrm{Zn}_{1-x} \mathrm{~F}_{2}$ were grown at UCSB by the Bridgman method. The platelets were polished to form a wedge in order to avoid the interference between light reflected from the upper plane and that from the lower plane of the sample. The strain at the surfaces of the sample, due to polishing, was removed by etching it in $70{ }^{\circ} \mathrm{C} \mathrm{HCl}$ solution for 1 minute. The transition temperatures are $T_{N}=78 \mathrm{~K}, 72 \mathrm{~K}, 66 \mathrm{~K}, 54 \mathrm{~K}$, and $45 \mathrm{~K}$ for $x=0.99,0.91,0.84,0.68$, and 0.58 , respectively. The FIR spectra were measured with a bandwidth resolution of $0.1 \mathrm{~cm}^{-1}$ using a FT-IR spectrophotometer made by the Bruker company, Germany. We used a high pressure Hg lamp as a light source, a myler film with $23 \mu \mathrm{m}$ thickness as a beamsplitter, and a Si- bolometer as a detector. The temperature, $T$, of the sample was controlled using a helium cryostat from the Oxford Instruments, U. K. 


\section{EXPERIMENTAL RESULTS}

The $T$ dependence of the FIR spectra of $\mathrm{Fe}_{0.84} \mathrm{Zn}_{0.16} \mathrm{~F}_{2}$ is shown in Fig. 1 as an example. The main broad absorption peak line becomes broader and its position shifts gradually to the lower energy side as $T$ increases below $T_{N}$. Two smaller satellite peaks are visible at all temperatures. The higher energy one becomes broader as $T$ increases, while the lower energy one becomes sharper. Above $T_{N}$, no absorption is observed. This $T$ dependence of the FIR spectra is found to be common to all the samples investigated.

The concentration dependence of the FIR spectrum of $\mathrm{Fe}_{x} \mathrm{Zn}_{1-x} \mathrm{~F}_{2}$ at $5 \mathrm{~K}$ is shown in Fig. 2. A very sharp absorption at $52.6 \mathrm{~cm}^{-1}$ is observed for $\mathrm{Fe}_{0.99} \mathrm{Zn}_{0.01} \mathrm{~F}_{2}$. The frequency of this absorption is in very good agreement with that of the antiferromagnetic resonance in $\mathrm{FeF}_{2}\left(52.7 \pm 0.2 \mathrm{~cm}^{-1}\right)$ reported by Ohlman and Tinkham [6]. However, there is a small absorption in the spectrum of $\mathrm{Fe}_{0.99} \mathrm{Zn}_{0.01} \mathrm{~F}_{2}$ near $50 \mathrm{~cm}^{-1}$ which is not observed in the pure system. The absorption line of $\mathrm{Fe}_{0.91} \mathrm{Zn}_{0.09} \mathrm{~F}_{2}$ is much broader than that of $\mathrm{Fe}_{0.99} \mathrm{Zn}_{0.01} \mathrm{~F}_{2}$. The oscillation in the spectrum of $\mathrm{Fe}_{0.91} \mathrm{Zn}_{0.09} \mathrm{~F}_{2}$ is considered to be an interference effect because this platelet is thicker than others. With increasing dilution, the absorption line becomes broader and its position shifts to lower energy. Taking into account the higher resolution of the FIR data that allows the main peak to be resolved into the two higher energy peaks, the spectrum of $\mathrm{Fe}_{0.58} \mathrm{Zn}_{0.42} \mathrm{~F}_{2}$ is consistent with the $q=0$ spectrum of $\mathrm{Fe}_{0.59} \mathrm{Zn}_{0.41} \mathrm{~F}_{2}$ measured by neutron scattering [5], except for the small lower energy peak in the FIR spectrum.

\section{DISCUSSION AND CONCLUSIONS}

To analyze the FIR spectra of $\mathrm{Fe}_{x} \mathrm{Zn}_{1-x} \mathrm{~F}_{2}$, we approximate the magnetic excitations in this system as single ion ones, taking the exchange interactions between $\mathrm{Fe}^{2+}$ spins into account in the form of a molecular field. This should be a good approximation since the results of the magnetization measurements made in $\mathrm{Fe}_{x} \mathrm{Zn}_{1-x} \mathrm{~F}_{2}$ were successfully explained 
based on a localized spin flip model [7]. The non-diluted compound $\mathrm{FeF}_{2}$ has the rutile type crystal structure $D_{4 h}^{14}-P 4 / m n m$ 8,9]. The $\mathrm{Fe}^{2+}$ free ion has a $3 d^{6}$ configuration and the ground state is ${ }^{5} \mathrm{D}$. Each $\mathrm{Fe}^{2+}$ ion is surrounded by six $\mathrm{F}^{-}$ions forming an octahedron. The orbital state of the high spin $\mathrm{Fe}^{2+}$ ion in an octahedral environment is split into the doublet ${ }^{5} \mathrm{E}$ and the triplet ${ }^{5} \mathrm{~T}_{2}$ by the cubic field: ${ }^{5} \mathrm{~T}_{2}$ is the ground state and ${ }^{5} \mathrm{E}$ is lifted up by some $10,000 \mathrm{~cm}^{-1}$. The rhombic field removes all the orbital degeneracy of ${ }^{5} \mathrm{~T}_{2}: \mathrm{A}_{1 g}$ is the ground state separated from $\mathrm{B}_{1 g}$ and $\mathrm{B}_{2 g}$ by $\sim 1115 \mathrm{~cm}^{-1}$ and $\sim 2400 \mathrm{~cm}^{-1}$ respectively 10 . Spin-orbit effects are adequately treated by perturbation methods, the effective Hamiltonian pertaining to the lowest orbital state of a single $\mathrm{Fe}^{2+}$ ion being

$$
\mathcal{H}=-D S_{z}^{2}+E\left(S_{x}^{2}-S_{y}^{2}\right)
$$

where $z$ is taken parallel to the crystalline $c$ axis and $D$ and $E$ are the uniaxial and orthorhombic anisotropy constants. As is seen from Fig. 1, the absorption intensity of the main signal decreases with increasing $T$. This means that the signal originates in the transition from the ground state. So, we assign the signals to the transition, from the ground state to the lowest excitation state, of which probability is suggested to be most dominant by matrix calculation of Hamiltonian of Eq. 1. The excitation energy, $\Delta E$, for this transition to occur at $T=0$ is

$$
\Delta E=\Delta E_{l}+|J|
$$

where $\Delta E_{l}=3(D-E)+3 E^{2} / D$ is a ligand field and $J$ is an exchange interaction. Tinkham estimated that $D=7.3( \pm 0.7) \mathrm{cm}^{-1}$ and $E=0.70( \pm 0.04) \mathrm{cm}^{-1}$ from the analysis of the paramagnetic resonance of $\mathrm{Fe}^{2+}$ in $\mathrm{ZnF}_{2}$ [11]. Guggenheim et al. estimated $D=6.46(+0.29$, $-0.10) \mathrm{cm}^{-1}$ by inelastic neutron scattering in $\mathrm{FeF}_{2}$ [12].

The probability, $p\left(i, n_{i}\right)$ that $n_{i}$ magnetic ions occupy the $z_{i} i$ th nearest neighbor sites is given by, $\frac{z_{i} !}{n_{i} !\left(z_{i}-n_{i}\right) !} x^{n_{i}}(1-x)^{z_{i}-n_{i}}$, with $i>0$ and where $x$ is the concentration of the magnetic ion. The sum of the local exchange interaction, $J\left(n_{i}\right)=J\left(n_{1}, n_{2}, \cdots\right)$, and the probability that $J\left(n_{i}\right)$ is operative, $P\left(n_{i}\right)=P\left(n_{1}, n_{2}, \cdots\right)$, are described as $J\left(n_{i}\right)=\sum_{i} J_{i} n_{i}$ 
and $P\left(n_{i}\right)=\prod_{i} p\left(i, n_{i}\right)$, where $J_{i}$ describes the exchange interaction to the $i$ th nearest neighbor. In the calculation, we consider the exchange interactions up to the third nearest neighbors. The values of parameters used are as follows: $J_{1}=+0.048 \mathrm{~cm}^{-1}, J_{2}=-3.64$ $\mathrm{cm}^{-1}, J_{3}=-0.194 \mathrm{~cm}^{-1}$ [13], $z_{1}=2, z_{2}=8$, and $z_{3}=4$. Taking into account the intrinsic line width of the experimental spectra, we calculate the spectrum by replacing $J$ in Eq.

2 by the Gaussian distribution of the probability of the local exchange interaction. The absorption spectrum as a function of energy, $A(E)$, is written readily as

$$
A(E)=\int \frac{P\left(|J|-\Delta E_{l}\right)}{\sqrt{2 \pi}} \exp \left\{\frac{E-\left(|J|-\Delta E_{l}\right)}{2 \sigma^{2}}\right\} d|J|,
$$

where $\Delta E_{l}$ and the width of the Gaussian distribution, $\sigma$, are parameters depending on the iron concentration $x$. The concentration dependence of the FIR spectrum of $\mathrm{Fe}_{x} \mathrm{Zn}_{1-x} \mathrm{~F}_{2}$ calculated using Eq. 3 is shown in Fig. 3. These spectra are qualitatively in good agreement with the main peak in the experimental data shown in Fig. 2. The molecular field approximation does not reproduce the three peaks structure which was suggested from neutron scattering, but confirms the basics of the magnetic excitations of $\mathrm{Fe}_{x} \mathrm{Zn}_{1-x} \mathrm{~F}_{2}$.

In conclusion, we have studied the temperature dependence and concentration dependence of the FIR spectra of $\mathrm{Fe}_{x} \mathrm{Zn}_{1-x} \mathrm{~F}_{2}$. The overall width and position of the main cluster of peaks in each of the low temperature FIR spectra is reproduced qualitatively by a calculation based on a single-ion excitation model under a molecular field with the distribution of the local exchange interactions. The detailed structure is, however, not adequately explained and further theoretical work is warranted. In particular, the positions of the resolved multiple FIR peaks are not reproduced by the model, consistent with conclusions of the previous neutron scattering study [5] at $x=0.59$.

\section{ACKNOWLEDGMENTS}

We would like to thank A. Fukaya, T. Mutou and K. Hashi for helpful discussions. This work was supported by the MR Science Research Program of RIKEN and DOE Grant 
No. DE-FG03-87ER45324. One of the present authors (J. S.) is supported by the Special Postdoctoral Researchers Program from RIKEN. 


\section{REFERENCES}

[1] D. P. Belanger, in Spin Glasses and Random Fields, edited by A. P. Young (World Scientific, Singapore, 1998) and references therein.

[2] M. F. Sykes and J. W. Essam, Phys. Rev. 133, A310 (1964);

[3] J. Satooka and A. Ito, J. Phys. Soc. Jpn. 66, 784 (1997);

[4] J. Satooka and A. Ito, J. Phys.: Cond. Mat. 10 L711 (1998).

[5] C. Paduani, D. P. Belanger, J. Wang, S-J. Han, and R. M. Nicklow, Phys. Rev. B 50, $193(1994)$.

[6] R. C. Ohlman and M. Tinkham, Phys. Rev. 123, 425 (1961).

[7] A. R. King, V. Jaccarino, T. Sakakibara, M. Motokawa and M. Date, Phys. Rev. Lett. 47, 117 (1981).

[8] J. W. Stout and S. A. Reed, J. Am. Chem. Soc. 76, 5279 (1954).

[9] J. W. Stout and E. Catalano, J. Chem. Phys. 23, 2013 (1955).

[10] J. W. Stout, M. I. Steinfeld, and M. Yuzuki, J. Appl. Phys. 39, 1141 (1968).

[11] M. Tinkham, Proc. Phys. Soc. A, 236, 535 (1956).

[12] H. J. Guggenheim, M. T. Hutchings, and B. D. Rainford, J. Appl. Phys. 39, 1120 (1968).

[13] M. T. Hutchings, B. D. Rainford, and H. J. Guggenheim, J. Phys. C 3, 307 (1970).

[14] C. B. de Araujo, Phys. Rev. B 22, 266 (1980). 


\section{FIGURES}
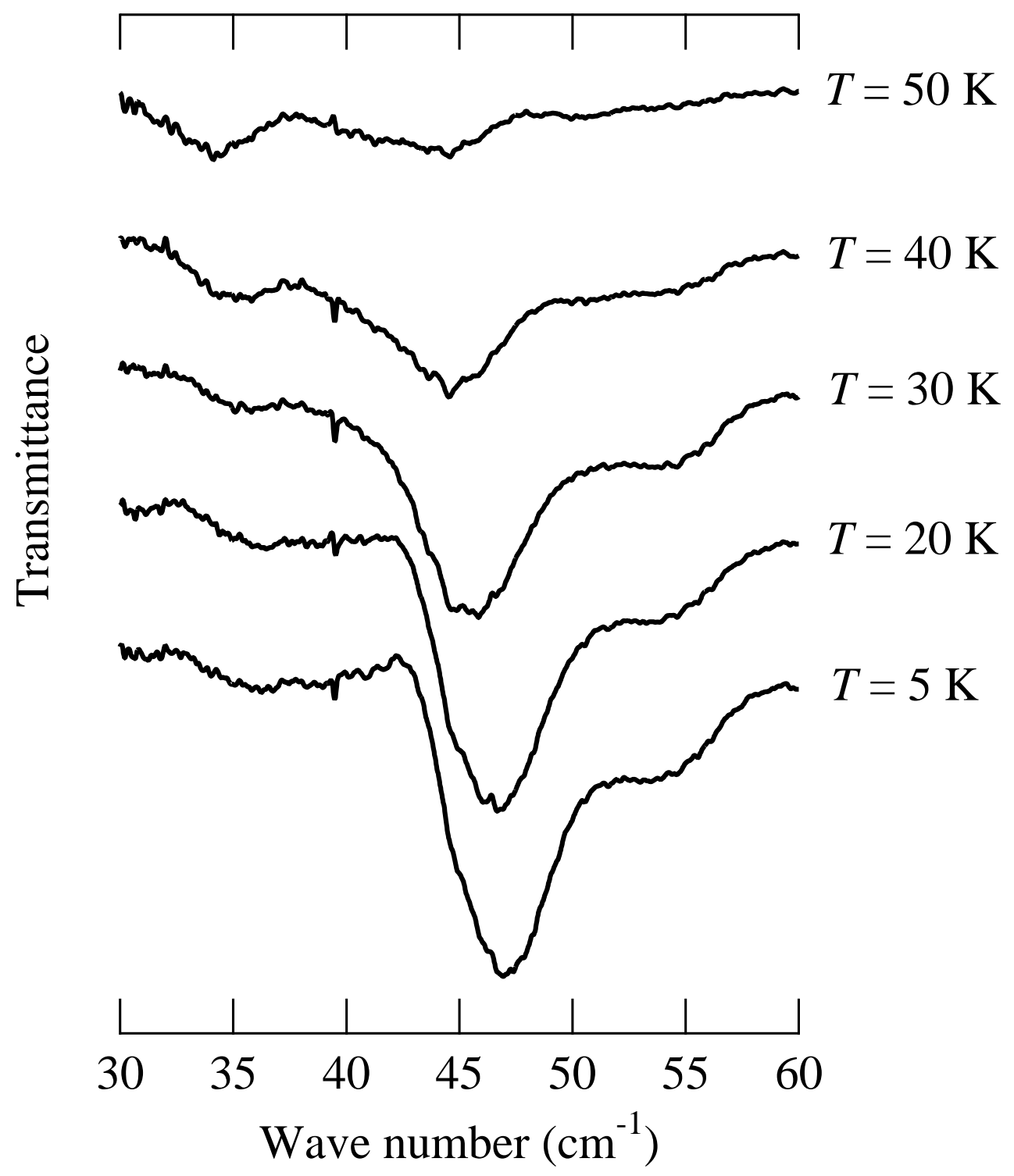

FIG. 1. Temperature dependence of the FIR spectrum of $\mathrm{Fe}_{0.84} \mathrm{Zn}_{0.16} \mathrm{~F}_{2}$. 


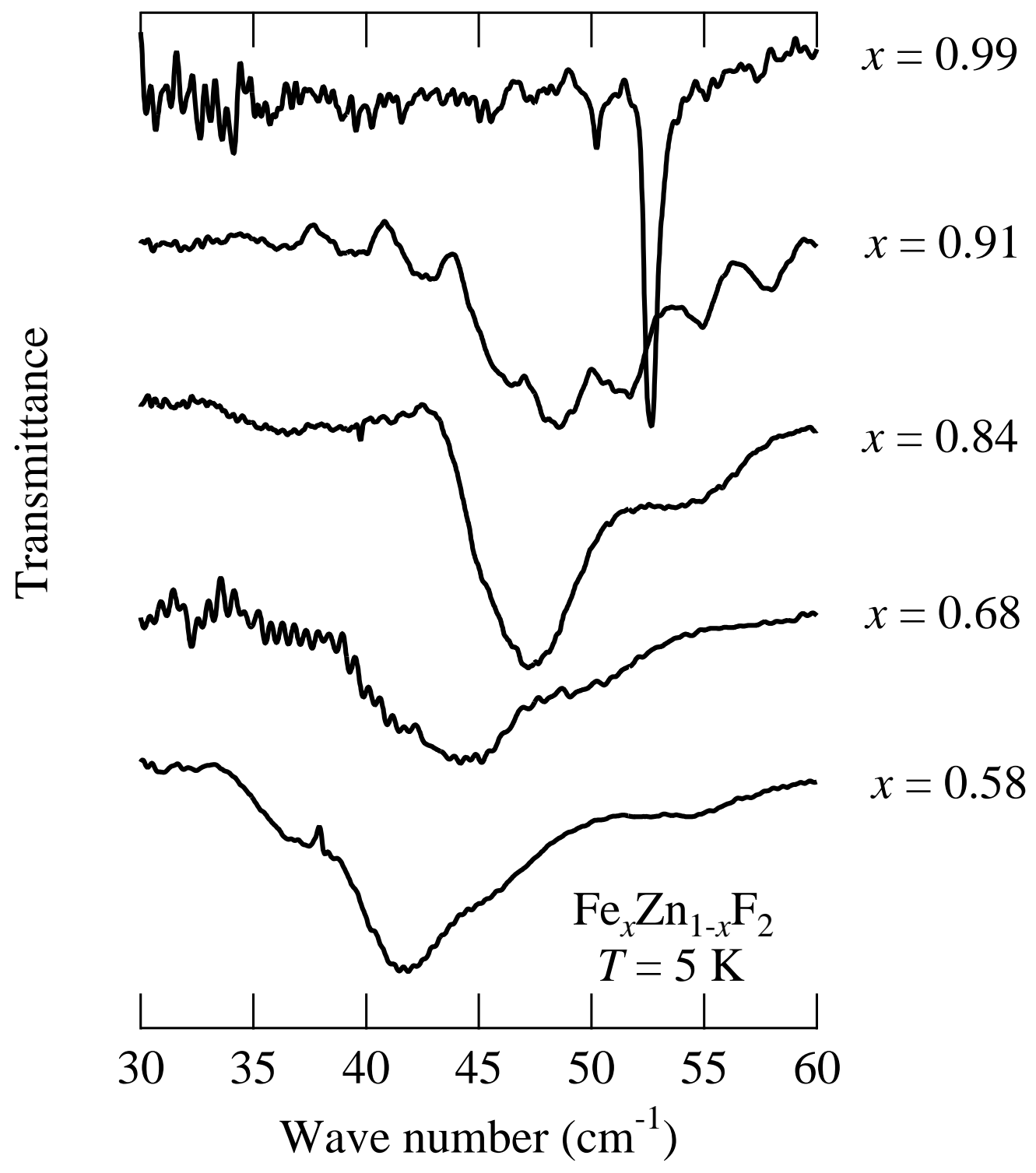

FIG. 2. Concentration dependence of the FIR spectrum of $\mathrm{Fe}_{x} \mathrm{Zn}_{1-x} \mathrm{~F}_{2}$ at $5 \mathrm{~K}$. 


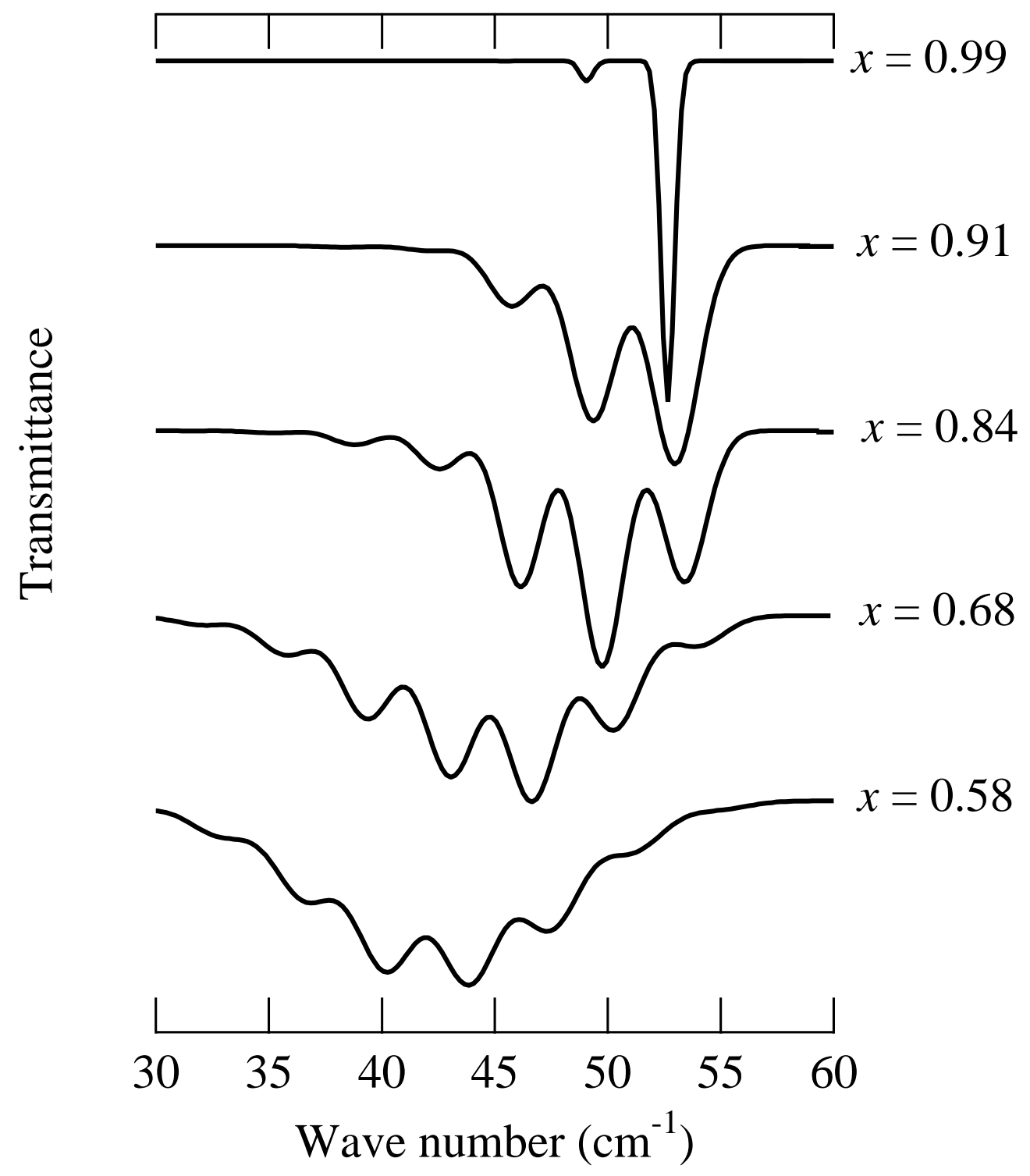

FIG. 3. Concentration dependence of the simulated FIR spectrum of $\mathrm{Fe}_{x} \mathrm{Zn}_{1-x} \mathrm{~F}_{2}$. 